\title{
The impact of the COVID-19 pandemic on urological care in Poland - Post-COVID resilience scenarios and recommendations for healthcare system: A national population-based modelling study.
}

\author{
Type \\ Research paper
}

\section{Keywords}

urology, Republic of Poland, COVID-19, pandemics, computer-aided modelling

\begin{abstract}
Introduction

Our aim was to assess the time required to recover the hypothetical surgical capacity of urological procedures that were suspended due to lockdowns caused by the SARS-CoV-2 outbreak in 2020 and 2021 in Poland, to indicate the most affected procedures, and to estimate the recovery time after a likely fourth lockdown.
\end{abstract}

\section{Material and methods}

The data aggregates contained the number of patients who underwent specific urological procedures classified in the ICD-9, performed from January 2009 to October 2019, acquired in granulation per month and per single voivodeship, and obtained by healthcare providers such as hospitals, ambulatory units, and primary care facilities. Using the model, we obtained the time required to discharge the excessive load on the healthcare system and the median wait time in the postlockdown period. We validated the model based on the data aggregates from March to October 2020.

\section{Results}

Leaving the capacity of the most affected procedures unaltered, or increasing it by $20 \%$, would not reduce the backlog of patients waiting to receive care after the third lockdown. The consequences of a feasible fourth lockdown would cause the necessity of a post-lockdown increase in capacity by more than $50 \%$.

\section{Conclusions}

The availability of the most affected procedures will never achieve the pre-pandemic state without increasing the hypothetical surgical capacity of urological procedures that were suspended due to lockdowns caused by the SARS-CoV-2 outbreak. These procedures require taking special steps to unblock the urological healthcare system and allow patients continuous access to treatment. 
The impact of the COVID-19 pandemic on urological care in Poland - PostCOVID resilience scenarios and recommendations for the healthcare system:

\section{A national population-based modelling study}

\section{Abstract}

Introduction: Our aim was to assess the time required to recover the hypothetical surgical capacity of urological procedures that were suspended due to lockdowns caused by the SARS-CoV-2 outbreak in 2020 and 2021 in Poland, to indicate the most affected procedures, and to estimate the recovery time after a likely fourth lockdown.

Materials and methods: The data aggregates contained the number of patients who underwent specific urological procedures classified in the ICD-9, performed from January 2009 to October 2019, acquired in granulation per month and per single voivodeship, and obtained by healthcare providers such as hospitals, ambulatory units, and primary care facilities. Using the model, we obtained the time required to discharge the excessive load on the healthcare system and the median wait time in the post-lockdown period. We validated the model based on the data aggregates from March to October 2020.

Results: Leaving the capacity of the most affected procedures unaltered, or increasing it by $20 \%$, would not reduce the backlog of patients waiting to receive care after the third lockdown. The consequences of a feasible fourth lockdown would cause the necessity of a post-lockdown increase in capacity by more than $50 \%$. 
Conclusion: The availability of the most affected procedures will never achieve the pre-pandemic state without increasing the hypothetical surgical capacity of urological procedures that were suspended due to lockdowns caused by the SARS-CoV-2 outbreak. These procedures require taking special steps to unblock the urological healthcare system and allow patients continuous access to treatment.

Keywords: COVID-19, pandemics, urology, Republic of Poland, computer-aided modelling 


\section{Introduction}

The COVID-19 outbreak has exposed major structural deficiencies in health, social, and economic policies and sectors [1-3]. In Poland, the disease has impacted the resilience of the healthcare system, further intensifying inequalities [4]. The vastly limited access to oncological and surgical services due to pandemic lockdowns has been especially challenging [5-7]. While policy-makers are often absorbed by the urgent day-to-day stresses of running a healthcare system, the COVID-19 pandemic has reminded everyone of the importance of resilience regaining tools, longer-term planning, and preparedness [8-10]. With this awareness comes the need to better understand health systems' strengths and vulnerabilities and how to respond resiliently to the outbreak, specifically in the face of the influenza season, economic impacts, and the potential resurgence of COVID-19 cases $[11,12]$.

Understanding the pathways of shocks induced by COVID-19 and their impact mechanism on urology among many healthcare subsectors can help policymakers prepare for diverse shocks and their fallout, and manage them better when they occur, including in other branches of medical activity. General healthcare system strengthening is critical for countries to help them prepare for and withstand wide-ranging shocks. In this connection, targeting particular weak areas may improve both system resilience and overall health system performance [13-15].

Our main focus was to estimate the burden of the SARS-CoV-2 pandemic on urological procedures and patient care in Poland $[4,16]$. The subsequent lockdowns caused a freeze of healthcare admissions in specialties other than 
COVID-19 and triggered an accumulation of patients requiring urgent surgical interventions. This work identifies the most affected procedures in urology based on a data modelling approach. These procedures require taking special measures to unblock the urological healthcare system and provide patients continuous access to treatment. We chose a regression model to assess the impact of the post-lockdown accumulation of patients requiring urological procedures, the duration of lockdown, and the maximum number of procedures that can be performed in a given month (hereinafter referred to as 'capacity').

Therefore, our aim was to assess the time required to recover the hypothetical capacity of urological procedures, with a special focus on surgical treatments-which were suspended due to three subsequent lockdowns in Poland caused by SARS-CoV-2 outbreaks in 2020 and 2021 -and to indicate the most critical cases. Our second objective was to estimate the healthcare system recovery time after a likely fourth lockdown in late 2021.

\section{Materials and Methods}

We obtained the data aggregates for the analysis from the Ministry of Health in Poland; they contained the number of patients who underwent specific urological surgical and diagnostic procedures from January 2009 to October 2019, and the median wait time for each procedure from March to October 2019 [17]. We acquired the data in granulation per month and per single voivodeship (we defined 'region' by NUTS2 methodology; regions are mostly coterminous with voivodeships) [18]. The database encompassed data aggregates from healthcare 
providers (i.e., hospices, long-term care units, hospitals, ambulatory units, and primary care facilities), which are obliged to report the number of procedures performed to the Polish National Health Fund for both inpatients and outpatients. To validate the model, we used the number of patients and the median wait time for each procedure from March to October 2020.

We determined the time required to discharge the excessive load on the healthcare system in the post-lockdown period using the regression methods for the urological procedures classified in the ICD-9 as follows: operations on the kidneys (system code: 55), operations on the ureter (56), operations on the urinary bladder (57), operations on the urethra (58), other operations on the urinary tract (59), operations on the prostate and seminal vesicles (60), operations on the scrotum and tunica vaginalis (61), operations on the spermatic cord, epididymis, and vas deferens (63), and operations on the penis (64) and their sub-procedures (both selective and urgent procedures).

We estimated the number of admitted patients from the regression model applied to the period from January 2009 to October 2019. The number of patients in the waiting queue (awaiting admission) was proportional to the product of median wait time and the number of admitted patients. The number of new patients in each month was the difference between the number of patients in the waiting queue in a given month and in a previous month.

From November 2019, we estimated the number of new patients with a minimisation model based on Powell's conjugate direction method [19, 20] and the following basis function: 
$y_{\text {new }}(t)=a * t+b+c * \sin (d * t+e)$,

where $y_{\text {new }}(\mathrm{t})$ - the number of new patients in month $t, a, b, c, d, e$ are the estimated parameters, $a{ }^{*} t+b-$ the regression component, and $\sin \left(d^{*} t+e\right)-$ the oscillatory component. We computed the numbers of patients in the waiting queue iteratively:

$y_{q}(t)=y_{q}(t-1)+y_{\text {new }}(t)-c(t) * y_{\text {admit }}(t)$,

where $y_{q}(t)$ - the number of patients in the waiting queue in month $t, y_{a d m i t}(t)-$ the number of admitted patients in month $t$, and $c(t)$ - maximal capacity in month $t$.

The urological procedures were partially suspended during the first lockdown from March to May 2020. The reduction in urological admissions (especially due to the transformation of medical service providers into COVID-19dedicated services) ranged from 11 to $91 \%$ depending on the procedure. During that period, maximal capacity—and thus the hypothetical number of procedures that would have been feasible to perform without the pandemic lockdown-were reduced. The number of patients in the waiting queue accounted for an additional load on the system after lockdown.

We then validated the model on the corresponding dataset for the period from March to October 2020. We assessed the differences between the estimated and actual numbers of admitted patients and median wait time using a two onesided equivalence t-test procedure, with the equivalence bounds in the range of half of the actual datasets for standard deviations. We checked the normality using the Kolmogorov-Smirnov normality test [21]. 
We assumed the second (November and December 2020) and third lockdowns' (February-April 2021) reduction in urological admissions and capacity values to be identical to the values during the first lockdown (March-May 2020). We assumed that in the month where the median wait time would be no higher than before the lockdowns, a pre-pandemic state would be reached [22]. The time spent (in months) between May 2021 and the month with a median not higher than that of February 2020 is the time required to recover the hypothetical surgical capacity of urological procedures that were suspended due to the three lockdowns caused by SARS-CoV-2.

We estimated the maximum monthly capacity for each procedure from the regression equation of the number of admitted patients. We assumed several model variants in which the healthcare system would operate with either the maximum estimated capacity, or with the estimated capacity increase from 10 to $100 \%$. A similar model can also be used to introduce what-if scenarios, addressing the prediction for an increase in capacity over a given time period to achieve the pre-lockdown state.

Because the pandemic outbreak has not yet finished and a fourth lockdown is highly probable, we simulated the hypothetical time required to recover the hypothetical surgical capacity if the fourth lockdown occurs from October to December 2021. During the analysis of recovery time after a feasible fourth lockdown, the time (in months) between January 2022 and the month with a median not higher than February 2020 would be the time required to recover the hypothetical surgical capacity of urological procedures. 


\section{Results}

The two one-sided t-tests showed that the estimated median wait time and estimated number of admitted patients from March to October were equivalent to actual values $(p<0.05)$ from March to October 2020, proving that the model was accurate. Detailed results are presented in Table I. Then, we applied the model to simulate the time (in months) required to recover the hypothetical surgical capacity to the pre-pandemic state after the third and expected fourth lockdown. The results for all procedures proved that the model produces significant results.

insert Table I here

Tables II and III summarize the time in months required to recover the hypothetical surgical capacity of the healthcare system when urological procedures were suspended during the third and fourth lockdowns. The tables outline the time periods for the entire country.

insert Table II here

insert Table III here

In conclusion, the most affected procedures were 63 operations on the spermatic cord, epididymis, and vas deferens, and 57 operations on the urinary bladder. The increase in capacity by $20 \%$ after the third lockdown will not ensure a reduction in wait time. We can assume that an approximately $14 \%$ increase means the extension of admissions by one working day. An increase of more than $10 \%$ requires more radical solutions to be performed and is presented in the discussion. Nevertheless, procedure 63 requires a more than $60 \%$ increase in capacity to 
avoid a consecutive rise in the number of waiting patients. Three lockdowns caused the accumulation of patients in the abovementioned procedures, which can be observed in the model. Lockdowns for 57 and 63 procedures caused increases in waiting for 39,072 and 1,252 patients, respectively.

We noted a slightly less severe situation for procedures 55 (operations on the kidneys), 56 (operations on the ureter), 58 (operations on the urethra), and 60 (operations on the prostate and seminal vesicles), which caused the accumulation of patients by $4,727,12,485,4,511$, and 14,532 people, respectively. An increase of $10 \%$ will most likely extend the wait time by more than 4 to 43 months. Hence, the total number of 90,363 patients may be affected by limiting all procedures during lockdowns. The large number of patients' overload might be due to diagnostic procedures being performed not only in hospitals, but also in primary care units and ambulatory care facilities.

We witnessed the best situation for 60 operations on prostate and seminal vesicles, and 61 operations on the scrotum and tunica vaginalis, for which the time to system recovery was less than three months without any increase in capacity.

The consequences of a feasible fourth lockdown will be serious for the most affected procedures unless special steps are taken. The post-lockdown increase in capacity for procedures 63 and 57 should be higher than $90 \%$ and $50 \%$ to avoid consecutive increases in wait time, respectively. Only such levels of increase would make it possible to achieve the pre-pandemic state in a finite amount of time. 
Figure 1 presents the plot of the relationship between the number of months needed to catch up to historical figures, and post-lockdown increases in procedure volumes. The potential what-if scenarios are feasible to set according to this plot; for example, to achieve the pre-lockdown state in 6 months, an increase in capacity should be provided for procedures $55,56,57,58,63$, and 64 by $32.5 \%$, $44.7 \%, 34.6 \%, 3.4 \%, 13.9 \%, 117.2 \%$, and $74.2 \%$, respectively (Figure 1). Figure 2 presents the same relationship, but takes the fourth hypothetical lockdown into account.

insert Figure 1 here

insert Figure 2 here

The time (in months) required to recover the hypothetical surgical capacity of the healthcare system can also be analysed per voivodeship. Figure 2 presents the time to recover for the most affected procedure, 56 , without increasing the maximum capacity $(0 \%)$, and from a $10 \%$ to $50 \%$ increase after the expected fourth lockdown. This example shows that the most affected voivodeship, due to delays in ureter surgeries, is West Lubusz Voivodeship (pl: woj. lubuskie) (Figure $3)$.

insert Figure 3 here

\section{Discussion}

The difficult situation related to the pandemic forced government-affiliated institutions to practically lock down healthcare admissions in specialties other than COVID-19. The lockdown caused the accumulation of patients requiring urgent 
surgical interventions. This work identifies the most affected procedures in urology for which the waiting queue of patients will never be reduced to the pre-pandemic state without an increase in the number of admissions and procedures. These procedures require special steps to be taken to avoid increasing the number of deaths caused by uro-oncological diagnosis. This research can be a milestone for creating a list of prioritised procedures that should be performed to unblock the urological healthcare system. It might be recommended to adopt criteria to prioritise procedures in Poland based on German, Spanish and Dutch case studies $[23,24]$, depending on the current epidemiological situation. Some authors and medical professional organisations created a list of prioritised interventions for rapidly progressing diseases [25-27]. Such actions, specifically for urological procedures in oncological diseases, may prevent numerous lives from being lost due to a lack of proper care [28-30].

Further, this research presents feasible what-if scenarios to predict an increase in capacity to unblock availability, thereby facilitating a return to the prepandemic state. The time required for the uro-oncological system to recover should be shorter than the time between COVID waves. Thus, recovery after the third lockdown should occur before expected SARS-CoV-2-related lockdowns. This can be achieved with systemic solutions taken at the government and local levels, such as the introduction of high-volume uro-oncological centres for the most specialised procedures, enhancing the capacity of primary healthcare units, and the widespread adoption of COVID-resilient methods of care, especially telemedicine [31-33]. Pandemic preparedness of the local healthcare system from a holistic 
perspective was inadequate. The local response to the spread of the coronavirus was too slow, and preparations to handle the pandemic were insufficient, which may be due to legislative and organisational chaos, especially during the first two waves of the pandemic infection surge. What-if scenarios, aimed at estimating a temporarily increased system load, ought to be introduced to soften the impact of epidemic incidents on the 'urological part' of the healthcare system in a finite time period. This approach seems to be even more appropriate to use in the upcoming waves of the pandemic, affecting health systems both locally and worldwide. A widely discussed, transparently communicated, and thoroughly explained what-if scenario strategy might serve as a beneficiary for the healthcare system, which is key given the morbidity of other-than-urological healthcare subsystems, especially cardiology- and oncology-related subsystems.

This paper can also be useful for local public administration representatives at the poviat (NUTS3) or voivodeship (NUTS2) levels, which are directly responsible for strategies against the spread of SARS-CoV-2. The decisions to close urological departments or transform them into COVID-dedicated departments can be supported by the presented model. The model proved to be valid. The equivalence test with two one-sided $t$ tests of simulated and real datasets indicated that the estimated values were accurate. Further research can be related to the extension of the results to a single hospital if relevant data become available. The expected outcomes may be more accurate with access to data, taking into account the actual hospital load in individual districts (poviats, voivodships), the number of 
available beds, operating theatres, and procedures performed in granulation for a single hospital.

The presented model can easily be applied to other medical specialties, not only for Poland, but also for other COVID-19-impacted countries.

\section{Conclusions}

In conclusion, the Polish healthcare system was able to adapt and scale-up on short notice for COVID-19-impacted patients needs'. Adaptation of the aforementioned healthcare system has been achieved at the price of extreme pressure on staff and of cancelled and severely postponed care. Hence, we are facing the gravity of the lasting consequences of the pandemic for a long time to come. This research presents the estimated time (in months) required to recover the hypothetical surgical capacity of urological procedures that were suspended due to lockdowns caused by the SARS-CoV-2 outbreak in 2020 and 2021 in Poland. The most affected procedures were 63 operations on the spermatic cord, epididymis, and vas deferens, and 57 operations on the urinary bladder, which required a more than $20 \%$ increase in capacity to avoid a consecutive rise in the number of waiting patients. The simulated fourth lockdown resulted in an additional increase in the patients' queue in an already overburdened healthcare system. It might be necessary to introduce appropriate legal measures in Poland, changing the scope of healthcare lockdown thresholds to limit the spread of the virus.

The post-fourth lockdown increase in capacity for procedures 63 and 57 should be higher than $90 \%$ and $50 \%$ to avoid consecutive increases in wait time, 
respectively. Special solutions, such as high-volume uro-oncological centres for the abovementioned prioritised procedures, should be performed. Moreover, what-if scenarios to estimate the increased load should be introduced to unblock the urological healthcare system in a finite time period (e.g., the increase in capacity should be $32.5 \%, 44.7 \%, 34.6 \%, 3.4 \%, 13.9 \%, 117.2 \%$, and $74.2 \%$ in procedures $55,56,57,58,63$, and 64 , respectively). This approach seems to be even more suitable for use in the upcoming waves of pandemics affecting health systems worldwide.

\section{Acknowledgements}

We would like to acknowledge the efforts of employees of from the Ministry of Health of the Republic of Poland for providing retrospective data required for the analysis, and the Ministry of Science and Higher Education in Poland for cofunding this research as a part of the "'Predictive Modeling of the COVID-19 Outbreak - ProMe"' project. We would like to extend our thanks to the General Statistical Office for having provided granular data on the Polish population. Special thanks go to the Ministry of Digital Affairs and Chancellery of the Prime Minister for their support in providing us with a complete death database. 


\section{References}

1. Miralles O, Sanchez-Rodriguez D, Marco E, et al. Unmet needs, health policies, and actions during the COVID-19 pandemic: a report from six European countries. Eur Geriatr Med 2021; 12: 193-204.

2. Wang C, Horby PW, Hayden FG, Gao GF. A novel coronavirus outbreak of global health concern. Lancet 2020; 395: 470-3.

3. Shanafelt T, Ripp J, Trockel M. Understanding and addressing sources of anxiety among health care professionals during the COVID-19 pandemic. JAMA 2020; 323: 2133-4.

4. Rogalski M, Dadel M, Sobkowska K, et al. COVID-19 data and analyses of infections, tests, deaths and cases recovered in Poland. https://docs.google.com/spreadsheets/d/1Tv6jKMUYdK6ws6SxxAsHVxZbgl ZfisC8x_HZ1jacmBM/edit\#gid=295642711. Accessed 15 May 2021.

5. Grasso AAC, Massa G, Castelnuovo M. The impact of COVID-19 pandemic on urological emergencies: a multicenter experience on over 3,000 patients. Urol Int 2021; 105: 17-20.

6. Bozkurt O, Sen V, Irer B, et al. Nation-wide analysis of the impact of Covid19 pandemic on daily urology practice in Turkey. Int J Clin Pract 2021; 75: e13735.

7. Ahmed K, Hayat S, Dasgupta P. Global challenges to urology practice during the COVID-19 pandemic. BJU Int 2020; 125: E5-6.

8. Hübner M, Zingg T, Martin D, Eckert P, Demartines N. Surgery for nonCovid-19 patients during the pandemic. PLoS One 2020; 15: e0241331. 
9. Tan $Y Q, W u Q H$, Chiong E. Preserving operational capability while building capacity during the COVID-19 pandemic: a tertiary urology centre's experience. Urology 2020; 142: 36-7.

10. Campi R, Amparore D, Capitanio U, et al. Assessing the burden of nondeferrable major uro-oncologic surgery to guide prioritisation strategies during the COVID-19 pandemic: insights from three italian high-volume referral centres. Eur Urol 2020; 78: 11-5.

11. Adebowale V, Alderson D, Burn W, et al. Covid-19: call for a rapid forward looking review of the UK's preparedness for a second wave-an open letter to the leaders of all UK political parties. BMJ 2020; 369: m2514.

12. Ficarra V, Novara G, Abrate A, et al. Urology practice during the COVID-19 pandemic. Minerva Urol Nefrol 2020; 72: 369-75.

13. Simonato A, Giannarini G, Abrate A, et al. Clinical pathways for urology patients during the COVID-19 pandemic. Minerva Urol Nefrol 2020; 72: 37683.

14. Chan MC, Yeo SEK, Chong YL, Lee YM. Stepping forward: urologists' efforts during the COVID-19 outbreak in Singapore. Eur Urol 2020; 78: e389.

15. Kontis V, Bennett JE, Rashid T, et al. Magnitude, demographics and dynamics of the effect of the first wave of the COVID-19 pandemic on allcause mortality in 21 industrialized countries. Nat Med 2020; 26: 1919-28. 
16. Sterne JA, White IR, Carlin JB, et al. Multiple imputation for missing data in epidemiological and clinical research: potential and pitfalls. BMJ 2009; 338: b2393.

17. Ministry of Health Analysis. https://analizy.mz.gov.pl/html/urologia_vs_covid19/. Accessed 15 May 2021.

18. Gerejczyk K, Pilewicz T. Special demographic zone in Poland - in search for the gist of the phenomenon. J Econ Manag 2017; 29: 37-57.

19. Powell MJ. An efficient method for finding the minimum of a function of several variables without calculating derivatives. Comput J 1964; 7: 155-62.

20. Arouxét M, Echebest N, Pilotta EA. Active-set strategy in Powell's method for optimization without derivatives. Comput Appl Math 2011; 30: 171-96.

21. Marsaglia G, Tsang WW, Wang J. Evaluating Kolmogorov's distribution. J Stat Softw 2003; 8: 1-4.

22. Medhi J. Stochastic Models in Queueing Theory. Amsterdam: Academic Press; 2003.

23. Paffenholz P, Peine A, Fischer N, et al. Impact of the COVID-19 pandemic on urologists in Germany. Eur Urol Focus 2020; 6: 1111-9.

24. Seguí-Moya E, González-Padilla DA, Ortega-Polledo LE, et al. Impact of COVID-19 in spanish urology residents: recommendations and perspective. Arch Esp Urol 2020; 73: 471-8.

25. Lai JW, Cheong KH. Superposition of COVID-19 waves, anticipating a sustained wave, and lessons for the future. Bioessays 2020; 42: e2000178. 
26. Naspro R, Da Pozzo LF. Urology in the time of corona. Nat Rev Urol 2020; 17: 251-3.

27. Nowroozi A, Amini E. Urology practice in the time of COVID-19. Urol J 2020; 17: 326 .

28. Quaquarini E, Saltalamacchia G, Presti D, et al. Impact of COVID-19 outbreak on cancer patient care and treatment: data from an outpatient oncology clinic in Lombardy (Italy). Cancers (Basel) 2020; 12: 2941.

29. Tachibana I, Ferguson EL, Mahenthiran A, et al. Delaying cancer cases in urology during COVID-19: review of the literature. J Urol 2020; 204: 926-33.

30. Ribal MJ, Cornford P, Briganti A, et al. European association of urology guidelines office rapid reaction group: an organisation-wide collaborative effort to adapt the European association of urology guidelines recommendations to the coronavirus disease 2019 era. Eur Urol 2020; 78 : $21-8$

31. Pang KH, Carrion DM, Rivas JG, et al. The impact of COVID-19 on European health care and urology trainees. Eur Urol 2020; 78: 6-8.

32. Proietti S, Gaboardi F, Giusti G. Endourological stone management in the era of the COVID-19. Eur Urol 2020; 78: 131-3.

33. Boehm K, Ziewers S, Brandt MP, et al. Telemedicine online visits in urology during the COVID-19 pandemic-potential, risk factors, and patients' perspective. Eur Urol 2020; 78: 16-20. 
Table I. Detailed method validation results of the estimated, actual, and mean differences between estimated and actual numbers of admitted patients, averaged per month and per procedure, as classified in the ICD-9. The results of the estimated, actual, and mean differences between the estimated and actual median wait time (in days) are averaged per month and per procedure, as classified in the ICD-9. The equivalence two-one sided tests are for $p$ values (non-inferiority and non-superiority checks) with a significance level $\alpha=0.05$. We performed validation of the entire country, and the model provided significant results.

\begin{tabular}{|cccccc|}
\hline $\begin{array}{c}\text { procedure } \\
\text { type } \\
\text { (ICD-9) }\end{array}$ & $\begin{array}{c}\text { estimated } \\
\text { average }\end{array}$ & $\begin{array}{c}\text { actual } \\
\text { average }\end{array}$ & $\begin{array}{c}\text { mean } \\
\text { difference }\end{array}$ & $\begin{array}{c}\text { non- } \\
\text { inferiority } \\
\text { p value }\end{array}$ & $\begin{array}{c}\text { non- } \\
\text { superiority } \\
\text { p value }\end{array}$ \\
\hline Number of admitted patients & & & & \\
\hline 55 & 2497 & 2482 & -15 & 0.0071 & 0.0151 \\
\hline 56 & 3629 & 3696 & 67 & 0.0335 & 0.0105 \\
\hline 57 & 14985 & 15131 & 146 & 0.0163 & 0.0059 \\
\hline 58 & 882 & 848 & -34 & 0.0006 & 0.0095 \\
\hline 59 & 822 & 832 & 10 & 0.0012 & 0.0005 \\
\hline 60 & 3910 & 3986 & 76 & 0.0367 & 0.0072 \\
\hline 61 & 317 & 297 & -21 & 0.0021 & 0.0428 \\
\hline 63 & 336 & 312 & -24 & 0.0001 & 0.0063 \\
\hline 64 & 1231 & 1124 & -107 & 0.0001 & 0.0311 \\
\hline Median wait time (days) & & & & & \\
\hline 55 & 33 & 32 & 0 & 0.0004 & 0.0097 \\
\hline 56 & 17 & 15 & -3 & 0.0406 & 0.0467 \\
\hline 57 & 143 & 146 & 2 & 0.0002 & 0.0001 \\
\hline 58 & 117 & 104 & -13 & 0.0014 & 0.0485 \\
\hline 59 & 38 & 36 & -2 & 0.0024 & 0.0253 \\
\hline 60 & 36 & 36 & 0 & 0.0290 & 0.0107 \\
\hline 61 & 43 & 40 & -3 & 0.0488 & 0.0193 \\
\hline 63 & 69 & 67 & -2 & 0.0059 & 0.0472 \\
\hline 64 & 44 & 43 & -1 & 0.0493 & 0.0112 \\
\hline
\end{tabular}


Table II. Time (in months) required to return the hypothetical surgical capacity to its pre-pandemic state in the healthcare system for the ICD-9's main procedures after three lockdowns. We performed the modelling without increasing the maximum capacity (0\%), and from a 10 to $100 \%$ increase.

\begin{tabular}{|c|c|c|c|c|c|c|c|c|c|c|c|}
\hline $\begin{array}{c}\text { procedure } \\
\text { type } \\
\text { (ICD-9) }\end{array}$ & $0 \%$ & $10 \%$ & $20 \%$ & $30 \%$ & $40 \%$ & $50 \%$ & $60 \%$ & $70 \%$ & $80 \%$ & $90 \%$ & $100 \%$ \\
\hline 55 & never & 32 & 10 & 6 & 4 & 3 & 3 & 2 & 2 & 2 & 1 \\
\hline 56 & never & 24 & 13 & 8 & 6 & 5 & 4 & 3 & 3 & 2 & 2 \\
\hline 57 & never & never never & 6 & 4 & 3 & 2 & 2 & 2 & 1 & 1 \\
\hline 58 & never & 4 & 3 & 2 & 2 & 1 & 1 & 1 & 1 & 1 & 1 \\
\hline 59 & 8 & 7 & 6 & 5 & 4 & 2 & 2 & 1 & 1 & 1 & 1 \\
\hline 60 & 2 & 1 & 1 & 1 & 1 & 0 & 0 & 0 & 0 & 0 & 0 \\
\hline 61 & 2 & 2 & 2 & 2 & 1 & 1 & 1 & 1 & 1 & 1 & 1 \\
\hline 63 & never & never never never & never & never & 17 & 11 & 9 & 7 & 6 \\
\hline 64 & never & 43 & 22 & 14 & 11 & 8 & 7 & 6 & 5 & 4 & 4 \\
\hline
\end{tabular}


Table III. Time (in months) required to return hypothetical surgical capacity to its prepandemic state in the healthcare system for the ICD-9's main procedures after all lockdowns, and a simulated one from October to December 2021 (the fourth lockdown). We performed the modelling without increasing the maximum capacity (0\%) and from a 10 to $100 \%$ increase.

\begin{tabular}{|r|c|c|c|c|c|c|c|c|c|c|c|}
\hline $\begin{array}{c}\text { procedure } \\
\text { type } \\
\text { (ICD-9) }\end{array}$ & $0 \%$ & $10 \%$ & $20 \%$ & $30 \%$ & $40 \%$ & $50 \%$ & $60 \%$ & $70 \%$ & $80 \%$ & $90 \%$ & $100 \%$ \\
\hline 55 & never & never & 16 & 10 & 7 & 5 & 4 & 3 & 3 & 3 & 2 \\
\hline 56 & never & 34 & 18 & 12 & 9 & 7 & 6 & 5 & 4 & 4 & 3 \\
\hline 57 & never & never & never & never & never & 7 & 5 & 4 & 3 & 2 & 2 \\
\hline 58 & never & never & 7 & 4 & 3 & 2 & 2 & 2 & 1 & 1 & 1 \\
\hline 59 & 16 & 12 & 9 & 7 & 6 & 5 & 5 & 4 & 4 & 3 & 3 \\
\hline 60 & 1 & 1 & 0 & 0 & 0 & 0 & 0 & 0 & 0 & 0 & 0 \\
\hline 61 & 1 & 1 & 0 & 0 & 0 & 0 & 0 & 0 & 0 & 0 & 0 \\
\hline 63 & never & never & never & never & never & never & never & never & never & 19 & 14 \\
\hline 64 & never & never & 31 & 21 & 16 & 13 & 10 & 9 & 8 & 7 & 6 \\
\hline
\end{tabular}




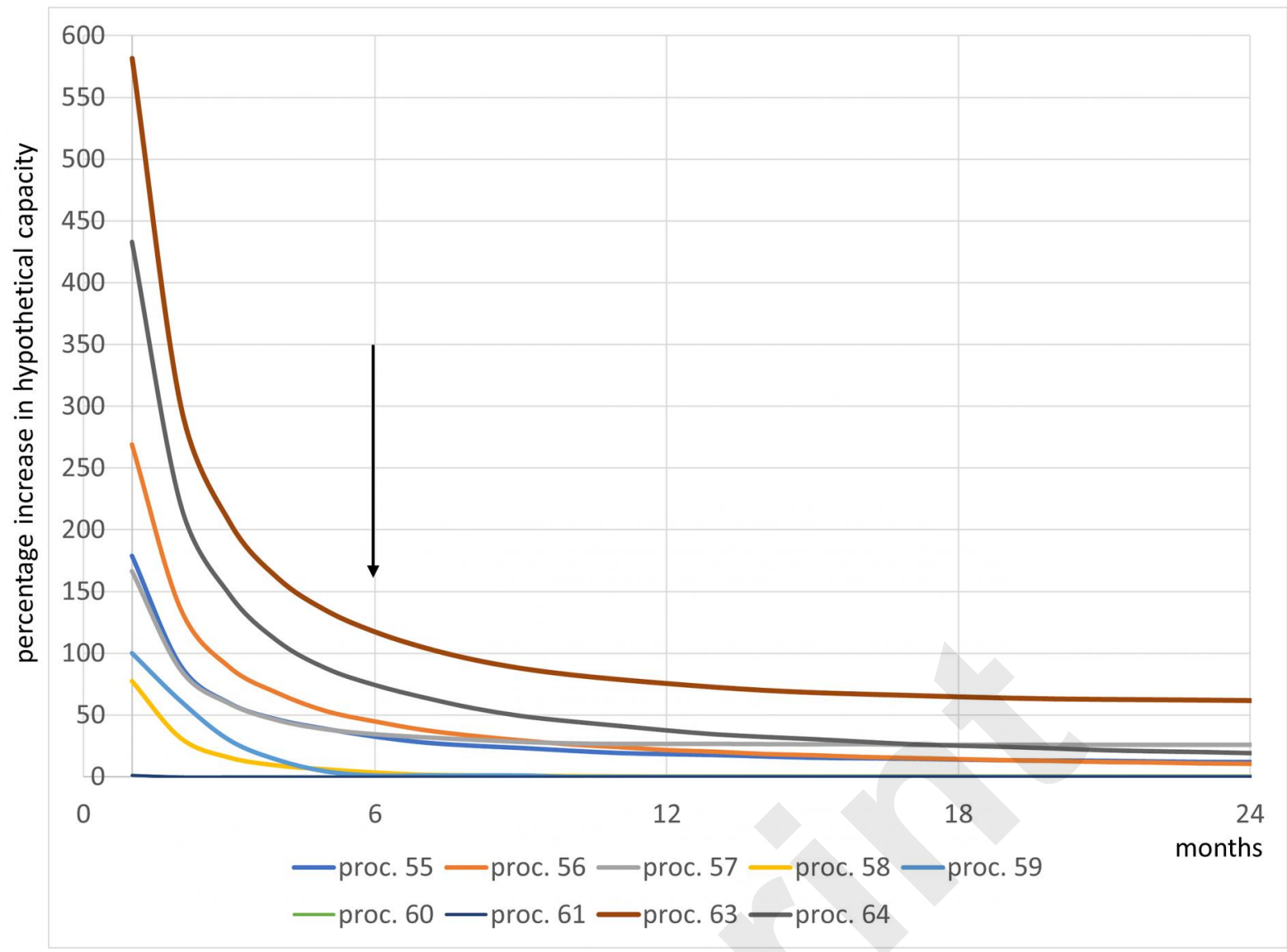

Plot of dependency between the time required to recover the hypothetical surgical volume of urological procedures (which were limited due to past lockdowns caused by the SARSCoV-2 outbreak in Poland) and the percentage increase in hypothetical capacity. The whatif scenarios can be easily set according to this plot; for example, if we assume that the prelockdown state is obtained after six months of resuming the patients' admissions (marked with a black arrow), then the minimum increase in capacity for each procedure can be determined from the ordinate axis. 


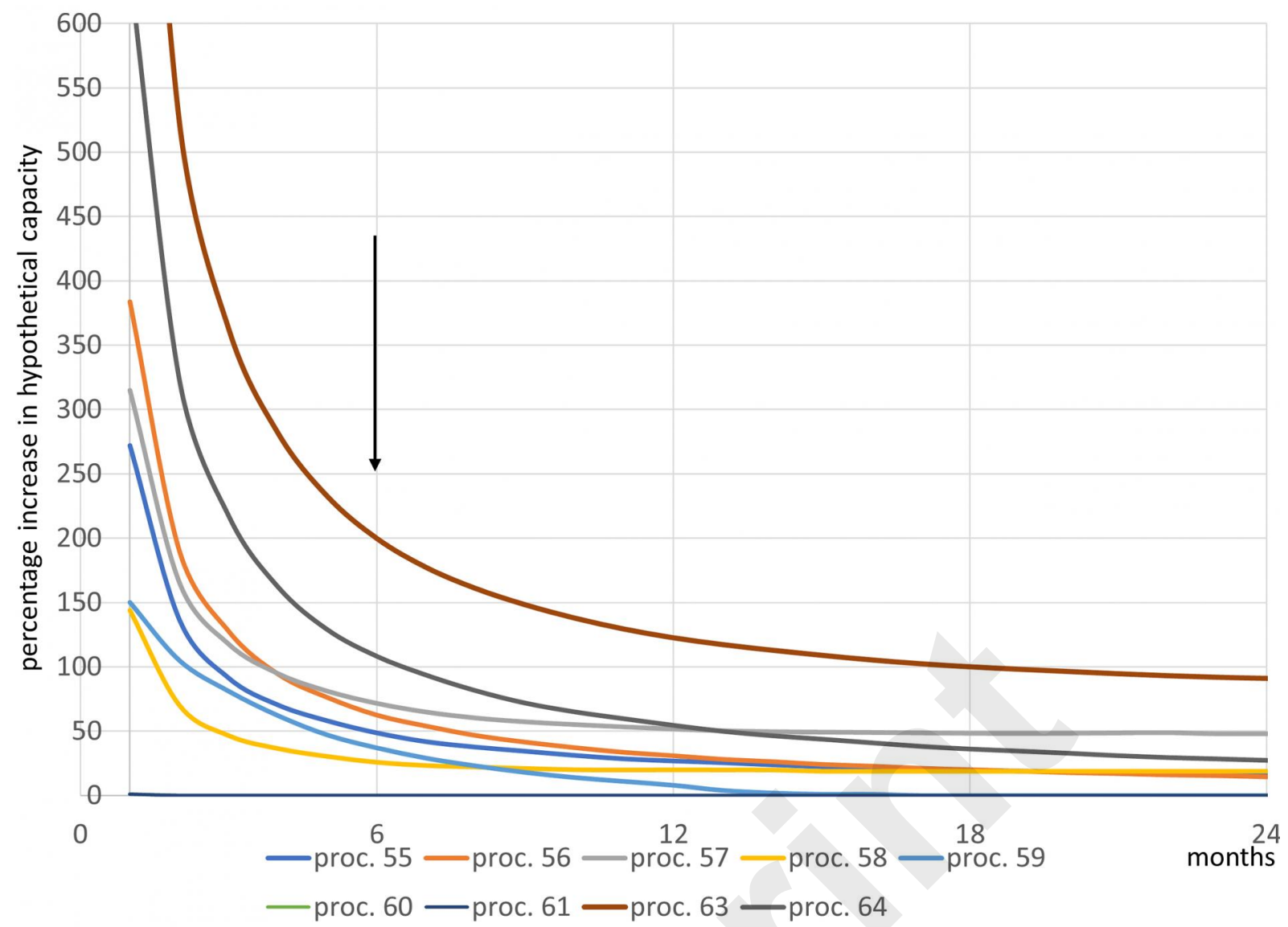

Plot of dependency between the time required to recover the hypothetical surgical volume of urological procedures, which were limited (and are going to be limited) after the fourth hypothetical lockdown. 

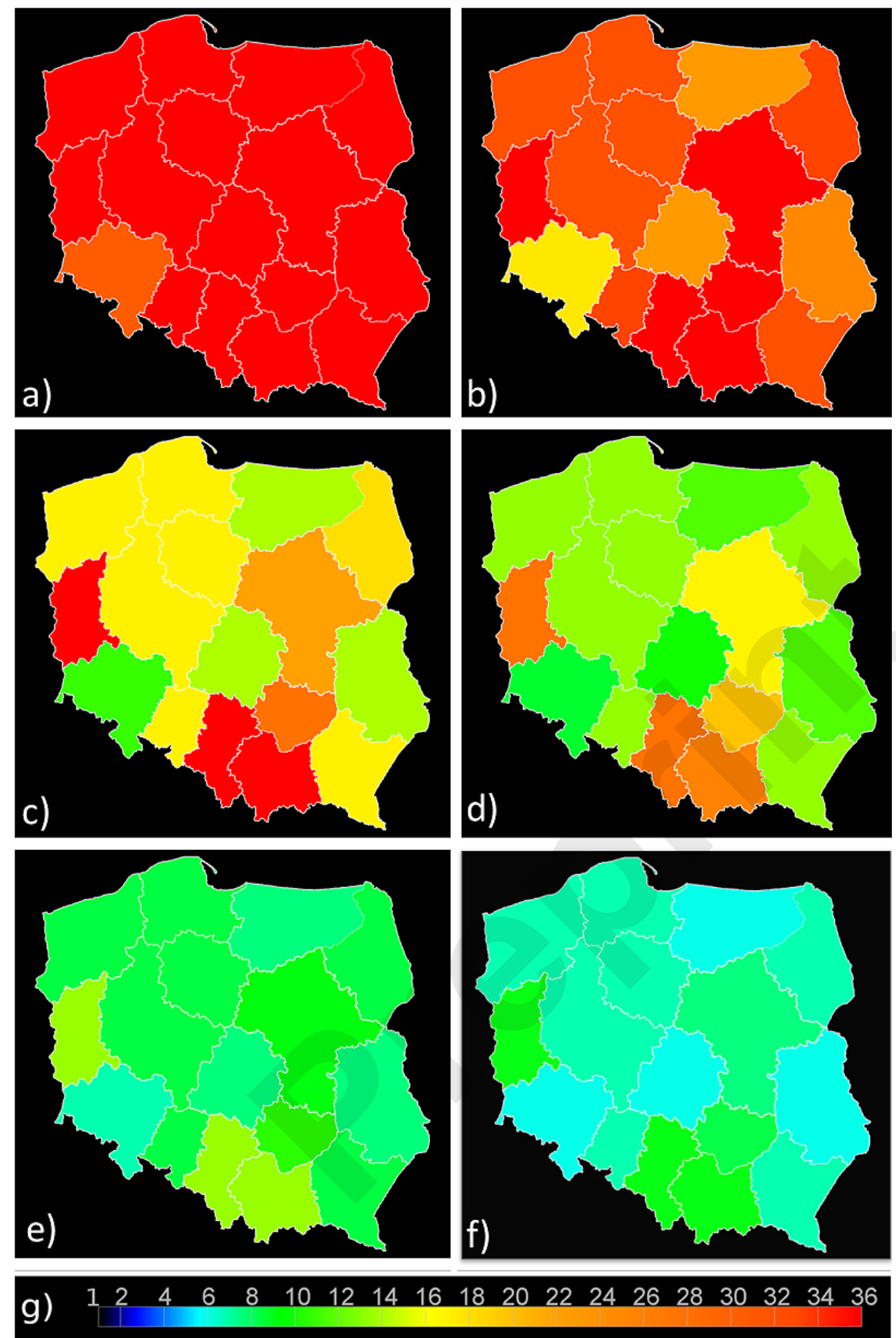

Time (in months) required to recover the hypothetical surgical capacity of the healthcare system for procedure 56 , operations on the ureter with (a) $0 \%$, (b) $10 \%$, (c) $20 \%$, (d) $30 \%$, (e) $40 \%$, and (f) $50 \%$ increase in capacity after the feasible fourth lockdown. Each voivodeship requires different recovery times. (g) To facilitate the assessment of the images, we used a time colormap. The voivodeships with time values over 36 months, or in which recovery is not possible, are marked in red. 